\title{
Modeling of Laser Induced Periodic Surface Structures
}

\author{
J. Z. P. Skolski ${ }^{1}$, G. R. B. E. Römer ${ }^{2}$, A. J. Huis in’t Veld ${ }^{2,3}$, V. S. Mitko ${ }^{1}$, J. V. Obona ${ }^{1,4}$, \\ V. Ocelik ${ }^{1,4}$, J. T. M. De Hosson ${ }^{4}$ \\ ${ }^{1}$ Materials innovation institute M2i, Mekelweg 2, Delft, The Netherlands \\ E-mail: j.skolski@m2i.nl \\ ${ }^{2}$ University of Twente, Faculty of Engineering Technology, Chair of Applied Laser Technology, \\ P.O. Box 217, 7500 AE, Enschede, the Netherlands \\ ${ }^{3}$ TNO Science \& Industry, Department Materials Technology, De Rondom 1, 5600 HE, \\ Eindhoven, The Netherlands \\ ${ }^{4}$ University of Groningen, Department of Applied Physics, Materials Science Centre and Netherlands \\ Institute of Metals Research, Nijenborgh 4, 9747 AG Groningen, The Netherlands
}

\begin{abstract}
In surfaces irradiated by short laser pulses, Laser Induced Periodic Surface Structures (LIPSS) have been observed on all kind of materials for over forty years. These LIPSS, also referred to as ripples, consist of wavy surfaces with periodicity equal or smaller than the wavelength of the laser radiation. Unfortunately, the physical phenomena explaining ripple initiation, growth and transitions toward other patterns are still not fully understood. Models, explaining ripple initiation and growth, based on the laser parameters, such as the wavelength and the angle of incidence, are frequently discussed in literature. This paper presents the most promising models, their ability and limitations to predict experimental results.

DOI:10.2961/jlmn.2010.03.0015
\end{abstract}

Keywords: Laser Induced Periodic Surface Structures, Ripples, Ultra-short laser pulses, Modeling

\section{Introduction}

Laser Induced Periodic Surface Structures (LIPSS) have been studied for forty five years and observed on many types of materials [1-4], however a complete understanding of their origin and growth is still missing. These structures, also referred to as ripples, are usually divided into Low Spatial Frequency LIPSS (LSFL) and High Spatial Frequency LIPSS (HSFL), see Figure 1. In this paper models are discussed, then a summary of the most promising, the efficacy factor theory, is presented. Eventually this theory along with a transient change of the complex refractive index is applied to explain LIPSS formation on alloyed steel [4].

\section{Models}

When created with a linearly polarized laser radiation at normal incidence, LSFL have a periodicity close to the laser wavelength $(\lambda)$ and a direction orthogonal to the polarization. They were observed for the first time by Birnbaum in 1965 and were attributed to a diffraction effect produced at the focus of a lens [1]. Several other explanations like frozen surface acoustic waves [5], plasma oscillations [6] or interference between the incident and scattered waves [7] were proposed during the 1970's. The influence of polarization, angle of incidence and wavelength of a laser beam on LIPSS strongly sustained the last assertion. In the 1980's, it was generally considered that LSFL arise from the interference process even though the nature of the surface scattered fields were still debated [8]. In 1983, Sipe et al. established a first principal theory for LIPSS formation, overcoming the physically inconsistent "surface-scattered wave" concept, by modeling the effect of surface roughness on the electromagnetic field [9]. A good agreement between experiments and theory was found by Young et al. [2], Clark and Emmony [10], and this for different laser parameters and materials. The theory of Sipe et al., also referred to as the efficacy factor theory or $\eta$ theory, is commonly accepted for the formation of LSFL. At normal incidence, LSFL produced with ultra short laser pulses have a periodicity smaller than the laser wavelength. Surface plasmon polaritons (SPP) have been recently proposed by several authors [11-15] as an explanation for the early stage formation of LSFL on semiconductors and dielectrics in the femtosecond regime. The optical properties of these materials change during the pulse due to the strong excitation, which gives semiconductors and dielectrics a metallic behavior. Therefore the ability to sustain SPP. The importance of SPP in LIPSS formation was already suggested by Keilmann and Bai in 1982 [16] and SPP excitation is included in the $\eta$ theory [9]. However the $\eta$ theory does not describe transient changes of the optical properties during a laser pulse. For this reason the excitation of SPP in the frame of this theory is confined to sufficiently conductive materials.

While LSFL can be obtained with either a CW laser or a pulsed laser, HSFL have only been observed for laser pulse durations in the picosecond or femtosecond range. For linearly polarized light at normal incidence, they have a periodicity much smaller than the laser wavelength and their direction can be parallel $[3,4]$ or orthogonal $[17,18]$ to the polarization, depending on the material and the laser parameters. The nature of HSFL is still debated and several theories have 


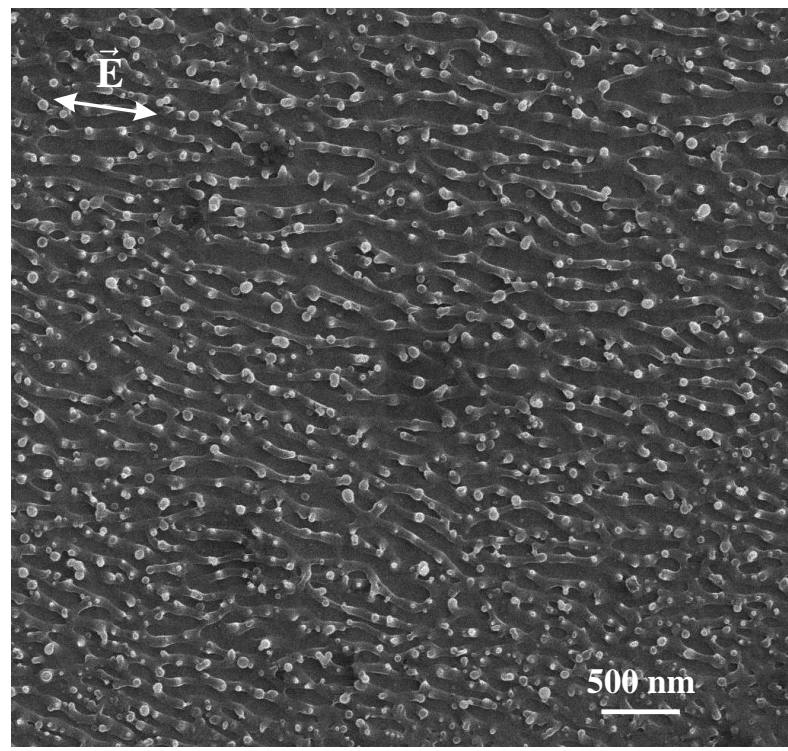

(a) HSFL parallel to the laser beam polarization with a periodicity $\Lambda_{H S F L} \approx 140 \mathrm{~nm}$. Nano bubbles, with diameters in the range $20-50 \mathrm{~nm}$, are preferentially found on the tops of HSFL

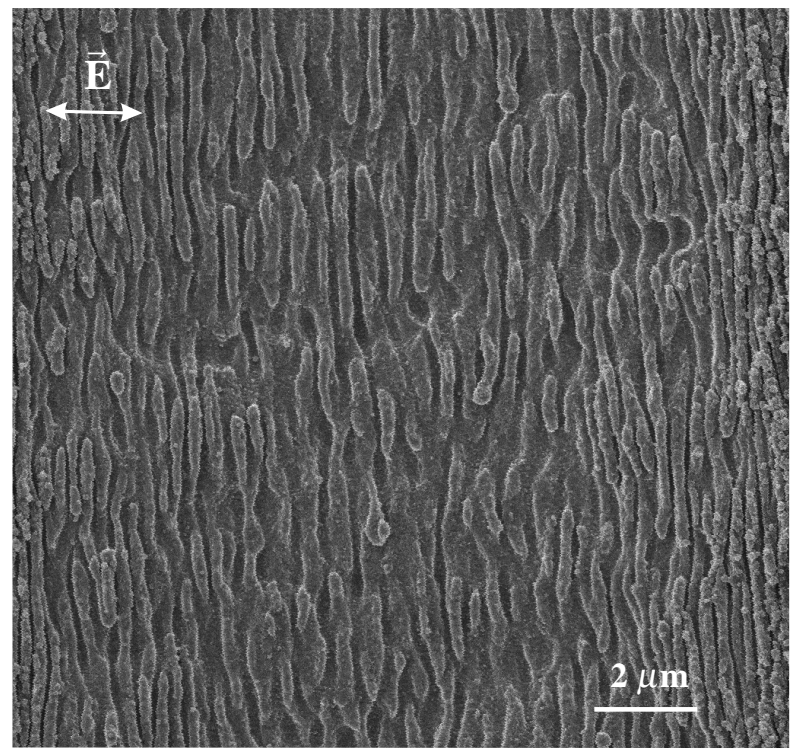

(b) LSFL orthogonal to the laser beam polarization with a periodicity $\Lambda_{L S F L} \approx 650 \mathrm{~nm}$ in the center of the image

Figure 1: Scanning Helium Ion Microscopy images of alloy $800 \mathrm{H}$ machined with $\lambda=800 \mathrm{~nm}, \theta=0$ and $E_{p}=40 \mathrm{~nJ}$.

been proposed to explain their formation: self organization $[19,20]$, second harmonic generation (SHG) $[17,21]$ or interference along with a modification of the optical properties during the pulse [3].

A theory considering the laser as a heat source, inducing a self organization process, will fail to explain the polarization dependency of HSFL. This dependence was a strong argument in favor of an interference approach to quantify LSFL periodicity, therefore a similar theory should be able to predict HSFL properties. The existence of HSFL only for ultra-short laser pulses, indicates that a non-equilibrium state of the matter should be taken into account in any modeling approach. That is why the periodicity predicted by a SHG theory using a constant refractive index cannot account for the HSFL [17]. Combining the efficacy factor theory, possible non-linear effects as SHG and a transient change in the material properties leads to fruitful conclusions. Wu et al. used the efficacy factor theory along with a modified refractive index to explain both the LSFL and the HSFL, parallel to the polarization, they obtained on diamond film [3]. It must be noticed that the efficacy factor theory was created to explain the LSFL formation, not the HSFL formation since these structures were not yet observed in the 1980's. Dufft et al. improved the approach of Wu et al. to account for the observed LIPSS on $\mathrm{ZnO}$ [18]. The transient change of the complex refractive index was modeled using the Drude model for different electron densities in the conduction band of the material, giving different efficacy factor graphs. SHG was also included by calculating the efficacy factor for half of the laser wavelength along with the changed refractive index. Good agreement was found for LSFL and HSFL, both orthogonal to the polarization.

\section{The efficacy factor theory}

Emmony et al. suggested in 1973 that LIPSS were a consequence of interference between the incident laser beam and surface-scattered waves [7]. Following this idea, Sipe et al. created the efficacy factor theory to account for LIPSS formation [9]. In the frame of this theory, three regions are defined in the $(\hat{x}, \hat{y}, \hat{z})$ space, where $\hat{x}=\vec{x} / x$ and $x$ is the norm of $\vec{x}$. As shown in Figure 2, for $z \geq 0$ there is vacuum and a region of thickness $l_{s}$, refered to as "selvedge", in which the roughness is confined. While, $z<0$ is the bulk material. The laser beam is modeled as an infinite plane wave of wavelength $\lambda, \vec{s}$ or $\vec{p}$ polarized, incident on the selvedge region at an angle of incidence $\theta$. The component of the wave vector parallel to the surface, the $(\vec{x}, \vec{y})$ plane, is referred to as $\vec{k}_{i}$. Instead of studying LIPSS formation in real space, with functions depending on $\vec{r}=(x, y, z)$, the process was studied in the Fourier domain, spanned by a wave vector $\vec{k}=\left(k_{x}, k_{y}\right)$ parallel to the surface. The goal of this approach is to predict the wave vector of the LIPSS, including their orientation and their periodicity $\Lambda=2 \pi / k$. The idea sustaining the Fourier domain calculations is that the diffraction patterns produced by a weak probe beam, illuminating a sample with LIPSS, are simple to understand in comparison to the observed structures in real space [8].

The laser beam striking the selvedge region creates scattered fields which interfere with the refracted field. This leads to an inhomogeneous energy absorption, just below the selvedge region, $A(\vec{k}) \propto \eta\left(\vec{k}, \vec{k}_{i}\right)|b(\vec{k})| . \quad \eta$ is called the efficacy factor and quantifies the efficacy with which the roughness leads to an inhomogeneous absorption at $\vec{k}$, while $b(\vec{k})$ is the Fourier component of the roughness. The main assumption is that LIPSS occur where $A(\vec{k})$ is the largest, hence $\eta(\vec{k})$ and $b(\vec{k})$ are governing their formation. To obtain these functions, $l_{s}$ is subject to two inequalities: 


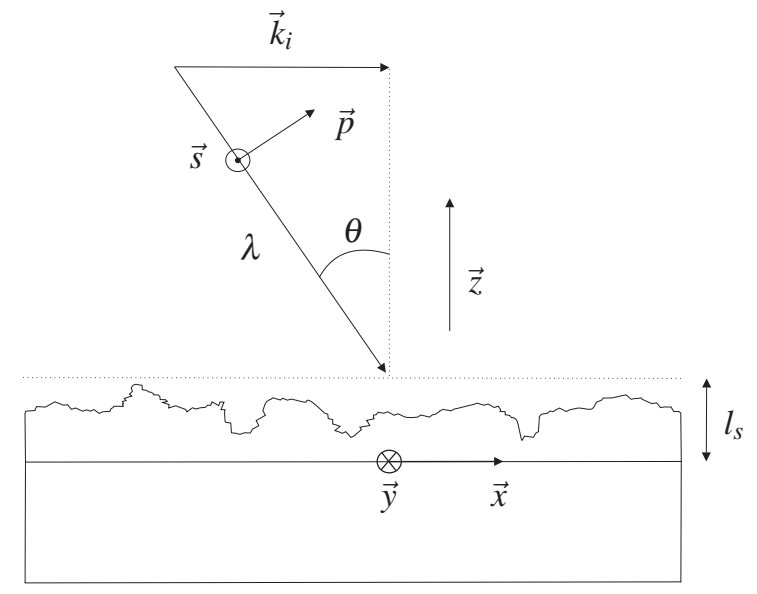

Figure 2: Geometry and notations used in the efficacy factor theory

$\tilde{\omega} l_{s} \ll 1, \quad k l_{s} \ll 1$,

That is, the selvedge thickness is small compared to the laser wavelength as well as to the possible LIPSS periodicity, $\tilde{\omega}=$ $2 \pi / \lambda$ being the norm of the laser wave vector. The function $b$ is defined in real space in this theory as a binary function introduced to describe the polarization $\vec{P}(\vec{r})$ in the selvedge:

$\vec{P}(\vec{r})=\chi b(\vec{r}) \vec{E}(\vec{r}) \quad\left(0<z<l_{s}\right)$,

where $\chi$ is the susceptibility of the bulk material and $b(\vec{r})=$ 1 or 0 respectively for the filled and unfilled parts of the selvedge. Instead of investigating the $b(\vec{r})$ function for each sample before irradiation, a more general approach has been followed by Sipe et al.. First, inequalities (1) lead to $b(\vec{r})=b(\vec{\rho})$, where $\vec{\rho}=(x, y)$. Next $b(\vec{\rho})$ is described in a probabilistic way by two parameters $F$ and $s$ which are respectively referred to as the filling factor and the shape factor:

$$
\left\{\begin{array}{l}
\langle b(\vec{\rho})\rangle=F \\
\left\langle b(\vec{\rho}) b\left(\vec{\rho}^{\prime}\right)\right\rangle=F^{2}+\left(F-F^{2}\right) C\left(\left\|\vec{\rho}-\vec{\rho}^{\prime}\right\|\right) \\
C(\vec{\rho})=\Theta\left(l_{t}-\|\vec{\rho}\|\right) \\
s=\frac{l_{t}}{l_{s}}
\end{array}\right.
$$

$F$ is the mean of the function $b(\vec{\rho}) . l_{t}$, and therefore $s$, characterizes how the filled part of $b(\vec{\rho})$ agglomerate through the $\left\langle b(\vec{\rho}) b\left(\vec{\rho}^{\prime}\right)\right\rangle$ expression. $\Theta$ is the unit step function. The best couple $(F, s)$ found to describe LIPSS equals $(0.1,0.4)$, which corresponds to spherically shaped islands [2], and is used for all the calculations in this paper.

The function $b(\vec{k})$ is expected to be a slowly varying function for a surface with homogeneously distributed roughness [9], while $\eta\left(\vec{k}, \vec{k}_{i}\right)$ has sharp peaks. When LIPSS start to grow, $b(\vec{k})$ changes to follow the peaks of $\eta\left(\vec{k}, \vec{k}_{i}\right)$, enhancing the absorption and the LIPSS formation. This qualitative feedback effect underlines that the driving function in LIPSS formation is $\eta\left(\vec{k}, \vec{k}_{i}\right)$. If $\lambda, \theta$, the polarization and the complex refractive $\tilde{n}$ of the material are known, and the couple
$(F, s)$ is set, it is possible to calculate $\eta\left(\vec{k}, \vec{k}_{i}\right)$ thanks to equation (4):

$$
\begin{aligned}
& \eta\left(\vec{k}_{,} \vec{k}_{i}\right)=2 \pi\left|v\left(\vec{k}_{+}\right)+v^{*}\left(\vec{k}_{-}\right)\right| \\
& \text {where } \vec{k}_{ \pm}=\vec{k}_{i} \pm \vec{k} \\
& v\left(\vec{k}_{ \pm}\right)=\left[h_{s s}\left(k_{ \pm}\right)\left(\hat{k}_{ \pm} \cdot \hat{x}\right)^{2}+h_{k k}\left(k_{ \pm}\right)\left(\hat{k}_{ \pm} \cdot \hat{y}\right)^{2}\right] \gamma_{t}\left|t_{s}\left(\vec{k}_{i}\right)\right|^{2}
\end{aligned}
$$

for s-polarized light and $\vec{k}_{i}$ parallel to $\hat{x}$. For p-polarized light

$$
\begin{aligned}
v\left(\vec{k}_{ \pm}\right)= & {\left[h_{s s}\left(k_{ \pm}\right)\left(\hat{k}_{ \pm} \cdot \hat{y}\right)^{2}+h_{k k}\left(k_{ \pm}\right)\left(\hat{k}_{ \pm} \cdot \hat{x}\right)^{2}\right] \gamma_{t}\left|t_{x}\left(\vec{k}_{i}\right)\right|^{2} } \\
& +h_{k z}\left(k_{ \pm}\right)\left(\hat{k}_{ \pm} \cdot \hat{x}\right) \gamma_{z} \varepsilon t_{x}^{*} t_{z} \\
& +h_{z k}\left(k_{ \pm}\right)\left(\hat{k}_{ \pm} \cdot \hat{x}\right) \gamma_{t} t_{z}^{*} t_{x} \\
& +h_{z z}\left(k_{ \pm}\right) \gamma_{z} \varepsilon\left|t_{z}\right|^{2}
\end{aligned}
$$

The $h, \gamma$ and $t$ functions can be found in the appendix.

The efficacy factor theory has several lacks and some of them were already pointed out by the authors [9]. Changes in the $b(\vec{k})$ function are not modeled, it is therefore impossible to use the $\eta$ theory on a pulse to pulse basis. Hence, the only possible quantitative predictions are related to the steady state LIPSS, governed by the efficacy factor. An already rippled surface can hardly be analyzed, since inequalities (1) are violated after the LSFL growth. Moreover, when described by the $(F, s)$ couple, $b(\vec{r})$ tends to be isotropic. The actual fluence applied during the laser irradiation, non-linear effects or high-order LIPSS [22] are not considered in the frame of the $\eta$ theory. Eventually, one of the main drawbacks is that the transient changes of the material properties during a laser pulse, and the influence of the pulse duration itself, are not taken into account. However, as stated in the second section, it is possible to partly overcome these problems and to use the efficacy factor to understand LSFL and HSFL formation [3, 18].

\section{Application of the theory}

The efficacy factor theory along with a transient change of the refractive index is used in this section to explain the results obtained by experiments on alloyed steel. For the sake of clarity, the relevant experimental parameters to understand the phenomena are summarized here. More information can be found in [4]. An alloy $800 \mathrm{H}$, an iron based alloy with $30 \%$ of nickel and $20 \%$ of chromium, was irradiated at normal incidence $\theta=0$ using a titanium sapphire based laser source with a central wavelength of $\lambda=800 \mathrm{~nm}$. The pulse duration was adjusted to $210 \mathrm{fs}$, the energy delivered per pulse on the sample was $E_{p}=40 \mathrm{~nJ}$ and the peak fluence was below the single pulse ablation treshold of alloyed steel. To avoid heat accumulation effects, the number of pulses $\mathrm{N}$ applied at the same location was changed by varying the number of overscans from 1 to 20 . For 1,2 and 5 pulses, only HSFL with a periodicity ranging from approximately 110 to $180 \mathrm{~nm}$ have been observed, while for 10 and 20 pulses both HSFL and LSFL, with a periodicity ranging from 234 to $238 \mathrm{~nm}$ and 620 to $714 \mathrm{~nm}$ respectively, have 


\begin{tabular}{cccccccc}
\hline Scans & $\begin{array}{c}\Lambda_{H S F L} \\
\text { in nm (Exp) }\end{array}$ & $\begin{array}{c}\Lambda_{L S F L} \\
\text { in nm (Exp) }\end{array}$ & Direct fit $\tilde{n}_{\text {eff }}$ & $\theta$ in degree & Polarization & $\begin{array}{c}\Lambda_{H S F L} \\
\text { in nm (theory) }\end{array}$ & $\begin{array}{c}\Lambda_{L S F L} \\
\text { in nm (theory) }\end{array}$ \\
\hline 1 & 111 & - & $7.5+0.2 i$ & 0 & $\vec{s}$ or $\vec{p}$ & 111 & - \\
2 & 140 & - & $6+0.2 i$ & 0 & $\vec{s}$ or $\vec{p}$ & 140 & - \\
5 & 180 & - & $4.7+0.2 i$ & 0 & $\vec{s}$ or $\vec{p}$ & 180 & - \\
10 & 234 & 714 & $3.6+0.13 i$ & 8 & $\vec{p}$ & 234 & 714 \\
20 & 238 & 620 & $3.6+0.13 i$ & 17.2 & $\vec{p}$ & 237 & 620 \\
\hline
\end{tabular}

Table 1: Periodicity of HSFL and LSFL

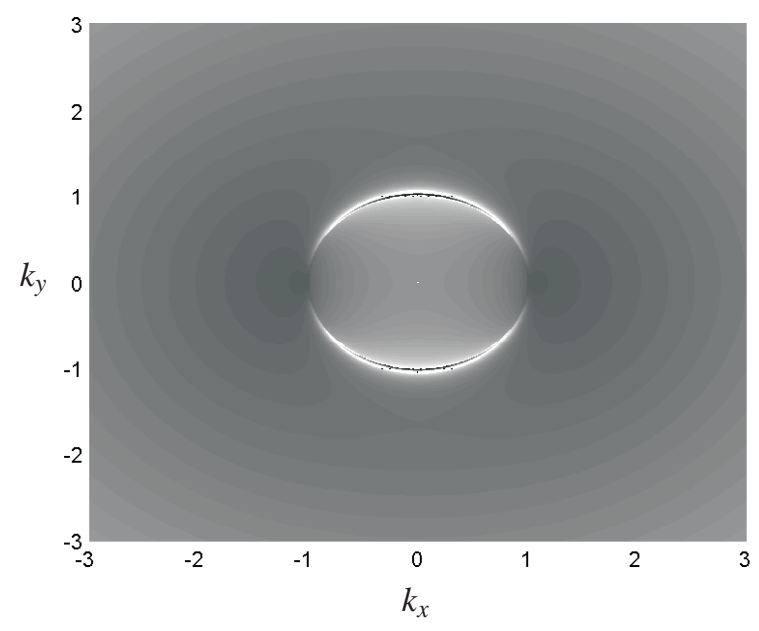

(a) $\tilde{n}=3.04+3.78 i$

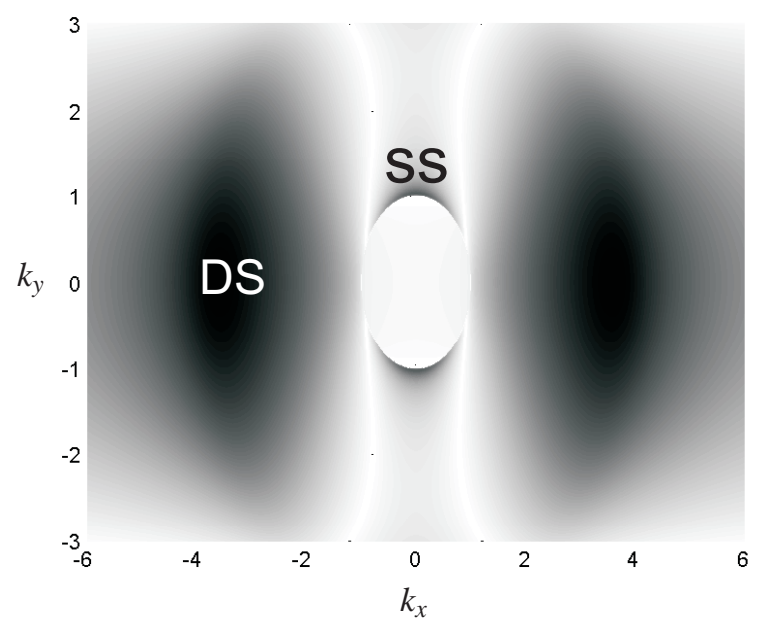

(b) $\tilde{n}_{\text {eff }}=4+0.5 i$

Figure 3: Gray scale 2D efficacy factor map for $\theta=0, \lambda=800 \mathrm{~nm}$ and different refractive indexes

been found. The different periodicities were calculated by applying a Fourier analysis of the Scanning Helium Ion Microscopy (SHIM) pictures and keeping only the frequencies with the largest magnitude. This approach is reproducible and allows a direct comparison of the experimental data with the $\eta$ theory. The results are summurized in table 1 .

The complex refractive index of the $800 \mathrm{H}$ alloy at $25^{\circ} \mathrm{C}$ and $\lambda=800 \mathrm{~nm}, \tilde{n}=3.04+3.78 i$, was estimated using a Drude model for alloys [23]. This standard value, obtained under the steady state and local thermal equilibrium assumptions, cannot explain the characteristics of the observed LIPSS since both conditions are violated. Figure 3(a) shows the 2D $\eta$ map for $\tilde{n}=3.04+3.78 i, \lambda=800 \mathrm{~nm}$ and $\theta=0$. It indicates only the presence of LSFL with a periodicity close to $\lambda$, in total disagreement with table 1 . This result is not surprising, as stated before, the $\eta$ theory takes one value of $\tilde{n}$ as an input while a function of time would be needed to model the transient behavior of this material property. If the variation of $\tilde{n}$ in time is known, the simplest approach to overcome this problem could be to draw several efficacy factor maps and calculate a peak power weighted average. In contrast to $\mathrm{ZnO}$ [18], a Drude model has failed to calculate the potential values taken by $\tilde{n}$ during the pulse for alloyed steel. Therefore, to account for the structures in the frame of the efficacy factor theory, an effective complex refractive index $\tilde{n}_{\text {eff }}$ is introduced here. It could be considered, in the best case, as a corrected $\tilde{n}$, which takes into account all the missing parameters of the theory, or ,in the worst case, as a meaningless parameter, which allows to test if the observed LIPSS are understandable in a purely electromagnetic approach. Two kind of structures in the $\eta$ maps, are relevant to explain the observed LIPSS. An example is shown in Figure 3(b). The well defined moon shape structures, referred to as type-s [2], are responsible for LSFL $\left(k_{x} \approx 0, \Delta k_{x} \approx 1, k_{y} \approx 1\right.$ and $\left.\Delta k_{y} \approx 0.5\right)$ while the darker areas $\left(k_{x} \approx 3.7, \Delta k_{x} \approx 2\right.$, $k_{y} \approx 0$ and $\Delta k_{y} \approx 2$ ) stands for HSFL. To our knowledge, only Wu et al. used these structures to explain the presence of HSFL on diamond [3]. These structures are special since they do not belong to the circles containing the usual type$s$ and type- $c$ structures [2]. They will be referred to as the dissident structures (DS) or type-d.

To understand the $\tilde{n}_{e f f}$ approach, few cross sections of 2D efficacy factor maps are shown in Figure 4. The DS (Figure 4(a)) and SS (Figure 4(b)) are not affected the same way by a change of $\tilde{n}_{e f f}$. A comparison of the solid and dash-dot lines shows that the imaginary part of $\tilde{n}_{e f f}$ governs the shape of both structures. If $\operatorname{Im}\left(\tilde{n}_{e f f}\right)$ decreases, the DS lose magnitude and spread, inducing a small shift of their maximum, while the SS are higher and sharper, therefore low and high $\operatorname{Im}\left(\tilde{n}_{e f f}\right)$ respectively favors DS and SS. It must be noticed that the absolute magnitude is not important, it is the difference of the absolute magnitude between DS and SS which is relevant. Comparing the solid and dashed lines shows that the real part of $\tilde{n}_{e f f}$ affects both the magnitude and the location of the DS. The larger $\operatorname{Re}\left(\tilde{n}_{e f f}\right)$ the further the location of the maximum of the DS is, hence large $\operatorname{Re}\left(\tilde{n}_{e f f}\right)$ should lead 


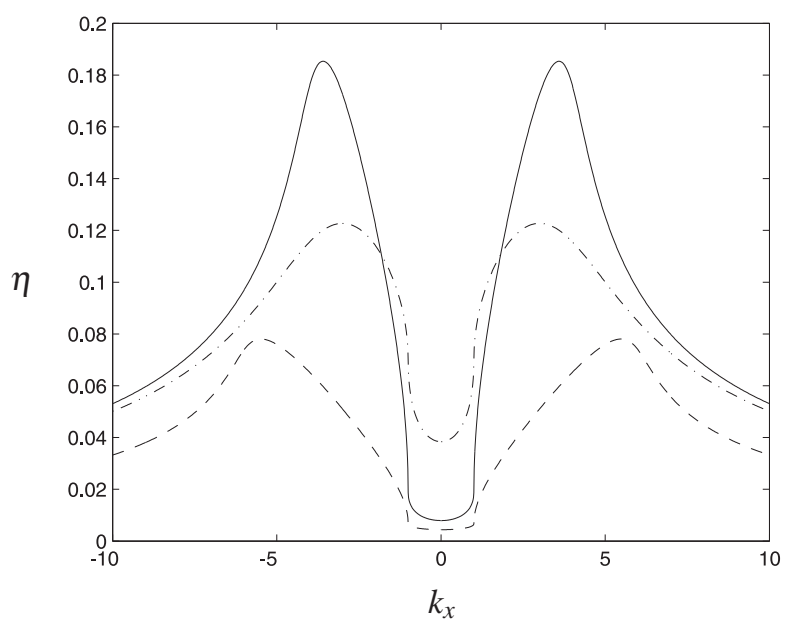

(a) Type- $d$ structure behavior

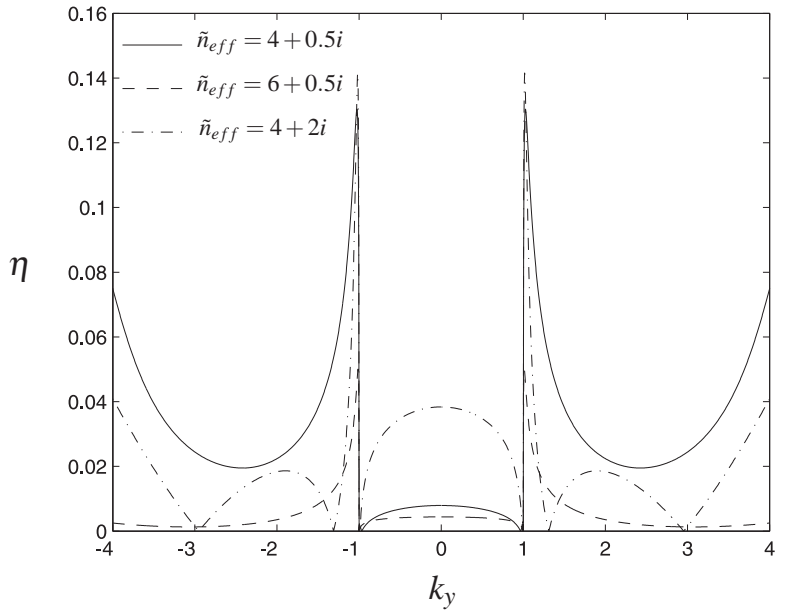

(b) Type-s structure behavior

Figure 4: Efficacy factor cross sections along $k_{x}$ and $k_{y}$ for $\theta=0, \lambda=800 \mathrm{~nm}, \tilde{n}_{e f f}=4+0.5 i$ (solid lines), $\tilde{n}_{e f f}=6+0.5 i$ (dashed lines) and $\tilde{n}_{e f f}=4+2 i$ (dash-dot lines).

to a small periodicity for the HSFL. The magnitude of the $\mathrm{SS}$ is also affected, but the difference of magnitude is almost constant, therefore $\operatorname{Re}\left(\tilde{n}_{e f f}\right)$ clearly governs the location of DS.

It is possible to choose an $\tilde{n}_{e f f}$ which makes a perfect match between the location of the DS and $\Lambda_{H S F L}$, as in the "direct fit $\tilde{n}_{\text {eff }}$ " column of table 1 . If these values are taken for $\tilde{n}_{\text {eff }}$ to understand $\tilde{n}$, it is not clear why the complex refractive index should have such a high real part, which decreases in function of the number of pulses, even if important phase changes between pulses are considered. $\tilde{n}_{e f f}$ is introduced here only to account for several effects: phase changes, the transient change of $\tilde{n}$ due to the laser excitation, but also the dynamics of the function $b(\vec{k})$ which is playing a significant role before the steady state patterns are developed. There is no reason to assume that $b(\vec{k})$ follows $\eta(\vec{k})$ prior to the first pulse. Assuming that the largest coefficients of $b(\vec{k})$ are not at the same $\vec{k}$ as the DS and SS, each pulse changes progressively the $b(\vec{k})$ function towards the maxima of the $\eta(\vec{k})$ function. Hence the periodicity of the HSFL will change on a pulse to pulse basis until the maximum of $b(\vec{k})$ and $\eta(\vec{k})$ match. That is why, even if it is possible to match perfectly the HSFL period and the DS by changing $\tilde{n}_{e f f}$, the values of the latter for $N=1,2$ and 5 do only partly reflect the variations of $\tilde{n}$. However it clearly shows that HSFL parallel to the polarization are a result of the DS, therefore understandable in the frame of an electromagnetic approach. To put in other words, the variation of $b(\vec{k})$ is neglected in the $\eta$ theory, therefore $\tilde{n}_{e f f}$ will not only reflect the transient changes of the optical properties but also the progressive change of $b(\vec{k})$, that is to say the modification of the surface roughness. By taking $\tilde{n}_{e f f}=3.6+0.13 i$, the periodicity of the steady states HSFL, obtained for $N \geq 10$, are in perfect agreement with the experimental values. For the sake of clarity, the efficacy factor cross sections for the $\tilde{n}_{\text {eff }}$ values in table 1 are not drawn on Figure 4 but the shape of the SS and DS looks similar to what is already presented. LSFL are also well described if a change of the angle of inci- dence due to the ablation process is considered. This change can be explained qualitatively by the formation of the crater as proposed by Wu et al. [3].

\section{Conclusion}

Both the HSFL and LSFL observed on alloyed steel can be understood in the frame of the efficacy factor theory, along with a change of the refractive index. More generally, LIPSS can be explained by an electromagnetic approach. LSFL are linked to the type-s and type-c fringes of the $\eta$ factor [2] while HSFL are divided into two categories: the ones orthogonal to the polarization, arising from type-s structures along with SHG as shown by Dufft et al. [18], and the ones parallel to the polarization, linked to the type- $d$ structures. No self organization seems to be required, or at least, it is not the driving phenomenon. The value of $\tilde{n}_{\text {eff }}=3.6+0.13 i$ strongly suggests that a general approach, as the $\eta$ theory, is better than trying to identify specific field structures. Quantitative predictions of LIPSS periodicity, width and height on a pulse to pulse basis are not possible yet. Indeed, the behavior of the inhomogeneous absorbed energy after the pulse, the exact variations of $\tilde{n}$ or the $b(\vec{k})$ function are not described by this theory. However the steady state HSFL and LSFL are quantitatively described if the correct $\tilde{n}_{e f f}$ is used. The effect of the fluence on LIPSS formation is still an open question.

\section{Acknowledgments}

This research was carried out under project number M61.3.08300 in the framework of the Research Program of the Materials innovation institute M2i (www.m2i.nl). 
Appendix

$$
\begin{aligned}
& h_{s s}\left(k_{ \pm}\right)=\frac{2 i \tilde{\omega}}{\sqrt{\tilde{\omega}^{2}-k_{ \pm}^{2}}+\sqrt{\tilde{\omega}^{2} \varepsilon-k_{ \pm}^{2}}} \\
& h_{k k}\left(k_{ \pm}\right)=\frac{2 i}{\tilde{\omega}} \frac{\sqrt{\tilde{\omega}^{2}-k_{ \pm}^{2}} \sqrt{\tilde{\omega}^{2} \varepsilon-k_{ \pm}^{2}}}{\varepsilon \sqrt{\tilde{\omega}^{2}-k_{ \pm}^{2}}+\sqrt{\tilde{\omega}^{2} \varepsilon-k_{ \pm}^{2}}} \\
& h_{z z}\left(k_{ \pm}\right)=\frac{2 i k_{ \pm}^{2}}{\varepsilon \sqrt{\tilde{\omega}^{2}-k_{ \pm}^{2}}+\sqrt{\tilde{\omega}^{2} \varepsilon-k_{ \pm}^{2}}} \\
& h_{z k}\left(k_{ \pm}\right)=\frac{2 i k_{ \pm}}{\tilde{\omega}} \frac{\sqrt{\tilde{\omega}^{2}-k_{ \pm}^{2}}}{\varepsilon \sqrt{\tilde{\omega}^{2}-k_{ \pm}^{2}}+\sqrt{\tilde{\omega}^{2} \varepsilon-k_{ \pm}^{2}}} \\
& h_{k z}\left(k_{ \pm}\right)=\frac{2 i k_{ \pm}}{\tilde{\omega}} \frac{\sqrt{\tilde{\omega}^{2} \varepsilon-k_{ \pm}^{2}}}{\varepsilon \sqrt{\tilde{\omega}^{2}-k_{ \pm}^{2}}+\sqrt{\tilde{\omega}^{2} \varepsilon-k_{ \pm}^{2}}} \\
& t_{s}\left(k_{i}\right)=\frac{2 \sqrt{\tilde{\omega}^{2}-k_{i}^{2}}}{\sqrt{\tilde{\omega}^{2}-k_{i}^{2}}+\sqrt{\tilde{\omega}^{2} \varepsilon-k_{i}^{2}}} \\
& t_{x}\left(k_{i}\right)=\frac{2}{\tilde{\omega}} \frac{\sqrt{\tilde{\omega}^{2}-k_{i}^{2}} \sqrt{\tilde{\omega}^{2} \varepsilon-k_{i}^{2}}}{\varepsilon \sqrt{\tilde{\omega}^{2}-k_{i}^{2}}+\sqrt{\tilde{\omega}^{2} \varepsilon-k_{i}^{2}}} \\
& t_{z}\left(k_{i}\right)=\frac{2}{\tilde{\omega}} \frac{k_{i} \sqrt{\tilde{\omega}^{2} \varepsilon-k_{i}^{2}}}{\varepsilon \sqrt{\tilde{\omega}^{2}-k_{i}^{2}}+\sqrt{\tilde{\omega}^{2} \varepsilon-k_{i}^{2}}} \\
& \gamma_{z}(F, s)=\frac{1}{4 \pi} \frac{\varepsilon-1}{\varepsilon-(1-F)(\varepsilon-1)\left(h(s)+R h_{i}(s)\right)} \\
& \gamma_{t}(F, s)=\frac{1}{4 \pi} \frac{\varepsilon-1}{1+\frac{1}{2}(1-F)(\varepsilon-1)\left(h(s)-R h_{i}(s)\right)} \\
& R=\frac{\varepsilon-1}{\varepsilon+1} \\
& h(s)=\left(s^{2}+1\right)^{\frac{1}{2}}-s \\
& h_{I}(s)=\frac{1}{2}\left[\left(s^{2}+4\right)^{\frac{1}{2}}+s\right]-\left(s^{2}+1\right)^{\frac{1}{2}}
\end{aligned}
$$

\section{References}

[1] M. Birnbaum. Journal of Applied Physics, 36:36883689, 1965.

[2] J. F. Young, J. S. Preston, H. M. van Driel, and J. E. Sipe. Physical Review B, 27:1155-1172, 1983.

[3] Q. Wu, Y. Ma, R. Fang, Y. Liao, Qi. Yu, X.i Chen, and K. Wang. Applied Physics Letters, 82:1703-1705, 2003.

[4] A. J. Huis in't Veld and H. van der Veer. JLMN-Journal of Laser Micro/Nanoengineering, 5:28-34, 2010.

[5] G. N. Maracas, G. L. Harris, C. A. Lee, and R. A. McFarlane. Applied Physics Letters, 33:453-455, 1978.
[6] N. R. Isenor. Applied Physics Letters, 31:148-150, 1977.

[7] D. C. Emmony, R. P. Howson, and L. J. Willis. Applied Physics Letters, 23:598-600, 1973.

[8] H. M. van Driel, J. E. Sipe, and J. F. Young. Physical Review Letters, 49:1955-1958, 1982.

[9] J. E. Sipe, J. F. Young, J. S. Preston, and H. M. van Driel. Physical Review B, 27:1141-1154, 1983.

[10] S. E. Clark and D. C. Emmony. Physical Review B, 40:2031-2041, 1989.

[11] A. Y. Vorobyev, V. S. Makin, and Chunlei Guo. Journal of Applied Physics, 101:034903-4, 2007.

[12] G. Martsinovskii, G. Shandybina, D. Smirnov, S. Zabotnov, L. Golovan, V. Timoshenko, and P. Kashkarov. Optics and Spectroscopy, 105:67-72, 2008.

[13] M. Huang, F. L. Zhao, Y. Cheng, N. Xu, and Z. Xu. ACS Nano, 3:4062-4070, 2009.

[14] E. Golosov, V. Emelyanov, A. Ionin, Yu Kolobov, S. Kudryashov, A. Ligachev, Yu Novoselov, L. Seleznev, and D. Sinitsyn. JETP letters, 90:107-110, 2009.

[15] J. Bonse, A. Rosenfeld, and J. Krüger. Journal of Applied Physics, 106, 2009.

[16] F. Keilmann and Y. H. Bai. Applied Physics A: Materials Science \& Processing, 29:9-18, 1982.

[17] A. Borowiec and H. K. Haugen. Applied Physics Letters, 82:4462-4464, 2003.

[18] D. Dufft, A. Rosenfeld, S. K. Das, R. Grunwald, and J. Bonse. Journal of Applied Physics, 105:034908-19, 2009.

[19] J. Reif, F. Costache, M. Henyk, and S. V. Pandelov. Applied Surface Science, 197-198:891-895, 2002.

[20] F. Costache, S. Kouteva-Arguirova, and J. Reif. Applied Physics A: Materials Science \& Processing, 79:1429-1432, 2004.

[21] J. Bonse, M. Munz, and H. Sturm. Journal of Applied Physics, 97:013538-9, 2005.

[22] P. M. Fauchet and A. E. Siegman. Applied Physics A: Materials Science \& Processing, 32:135-140, 1983.

[23] S. B. Boyden and Y. Zhang. Journal of Thermophysics and Heat Transfer, 20:9-15, 2006.

(Received: June 07, 2010, Accepted: November 22, 2010) 